\title{
Ascertainment of familial ovarian cancer in the Aberdeen Genetic Clinic
}

Helen Gregory, Andrew Schofield, Deepthi de Silva, June Semper, Benedict Milner, Lindsey Allan, Neva Haites

\begin{abstract}
Ovarian cancer is the fifth most common malignancy in women in the UK. Patients with a family history including ovarian cancer make up nearly $15 \%$ of family cancer referrals to the Genetic Clinic in Aberdeen. To date, only one pedigree has been suitable for linkage studies, which has enabled us to target screening more accurately at those people at highest risk. Following discovery of a strong candidate for the BRCA1 gene, direct mutation testing may soon be possible. People who seek testing will require further counselling. Therefore we anticipate an increased demand on both clinical and laboratory resources, but more accurate ascertainment of high risk subjects should lead to more appropriate targeting of screening services.

(f Med Genet 1996;33:187-192)
\end{abstract}

Key words: familial ovarian cancer; Aberdeen Genetic Clinic; BRCA1.

The annual incidence of ovarian cancer is about 15 per 100000 females in the $\mathrm{UK},{ }^{1}$ making it the fifth most common malignancy in females. Epithelial ovarian cancer is asymptomatic in the early stages and is hence often diagnosed at an advanced stage, perhaps explaining the poor prognosis and five year survival rate of less than $35 \%$. $^{2}$ Five to $10 \%$ of epithelial ovarian cancers occur in relation to an hereditary predisposition which has an autosomal dominant mode of transmission. ${ }^{34}$ In such families, the predisposition is most often to ovarian and breast cancer, although ovarian specific families have been documented.

For the purposes of this paper, a significant family history of ovarian cancer was defined as follows: (1) one or more first degree relatives with epithelial ovarian cancer under 55 years of age, (2) one or more first degree relatives with both breast and epithelial ovarian cancer at any age, (3) one first degree relative with epithelial ovarian cancer at any age and one or more additional first or second degree relative with breast or ovarian cancer, (4) one first degree relative with breast cancer and one or more additional first or second degree relatives with ovarian cancer at any age.

The present management of patients who present with a significant family history of ovarian cancer may include regular screening using serum CA125 estimation and pelvic ultrasound in addition to bimanual pelvic ex- amination. Prophylactic oophorectomy may be considered after completion of childbearing in those who are thought to be at high risk and who request this procedure following counselling. ${ }^{5}$ With the linkage of ovarian and breast/ovarian cancer predisposition to the BRCA1 gene on $17 \mathrm{q}$ and its recent cloning, ${ }^{67} \mathrm{DNA}$ tests to identify those at highest risk have become possible.

We have studied $3 \frac{1}{2}$ years of referrals to the Genetic Clinic in Aberdeen of people thought to be at high risk of developing cancer. From these referrals, we have ascertained those pedigrees containing a significant history of ovarian cancer as an estimate of the potential workload that could be produced once presymptomatic genetic testing (including mutation testing) becomes available. It should be noted that not all of these patients were referred primarily because of a history of ovarian cancer, for example, those with a history suggestive of a Lynch type II syndrome. We anticipate that there will be a significant increase in workload if current guidelines for pre- and post-test counselling are implemented. ${ }^{8}$

DNA linkage studies have been performed in one large family with a history of breast and ovarian cancers, using markers flanking and within BRCA1 on chromosome 17q. Currently the maximum lod score is 1.7 for the most informative loci using marker D17S579 (5\% recombination), but using an intragenic marker D17S855 the lod score is $1.523(0 \%$ recombination). The results have shown that the number of people considered to be at high risk and requiring screening or prophylactic surgery or both can be reduced, with consequent potential savings for the screening services, and the potential for reduction in anxiety for those family members found to be at low risk. Currently all at risk women are continuing to receive screening, pending the results of studies to identify a mutation in BRCA1.

\section{Method and results}

Between January 1990 and July 1994, 968 patients with a family history of cancer were referred for genetic counselling in Aberdeen. These were drawn from a population of around

\begin{tabular}{ll} 
Table $1 \quad$ Types of cancer family & \\
\hline Cancers reported & Pedigrees \\
\hline Ovarian only & 20 \\
Breast/ovarian & 25 \\
Ovarian and other (eg Lynch II) & 11 \\
Total & 56 \\
\hline
\end{tabular}


A

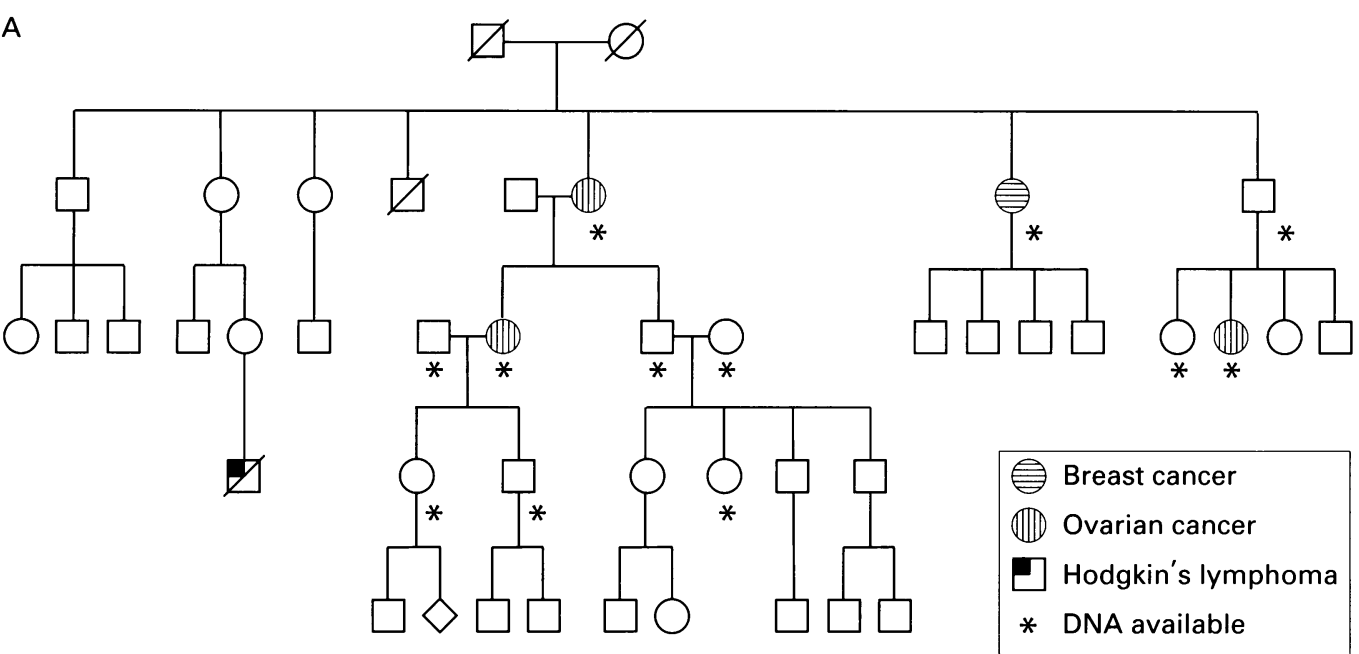

B

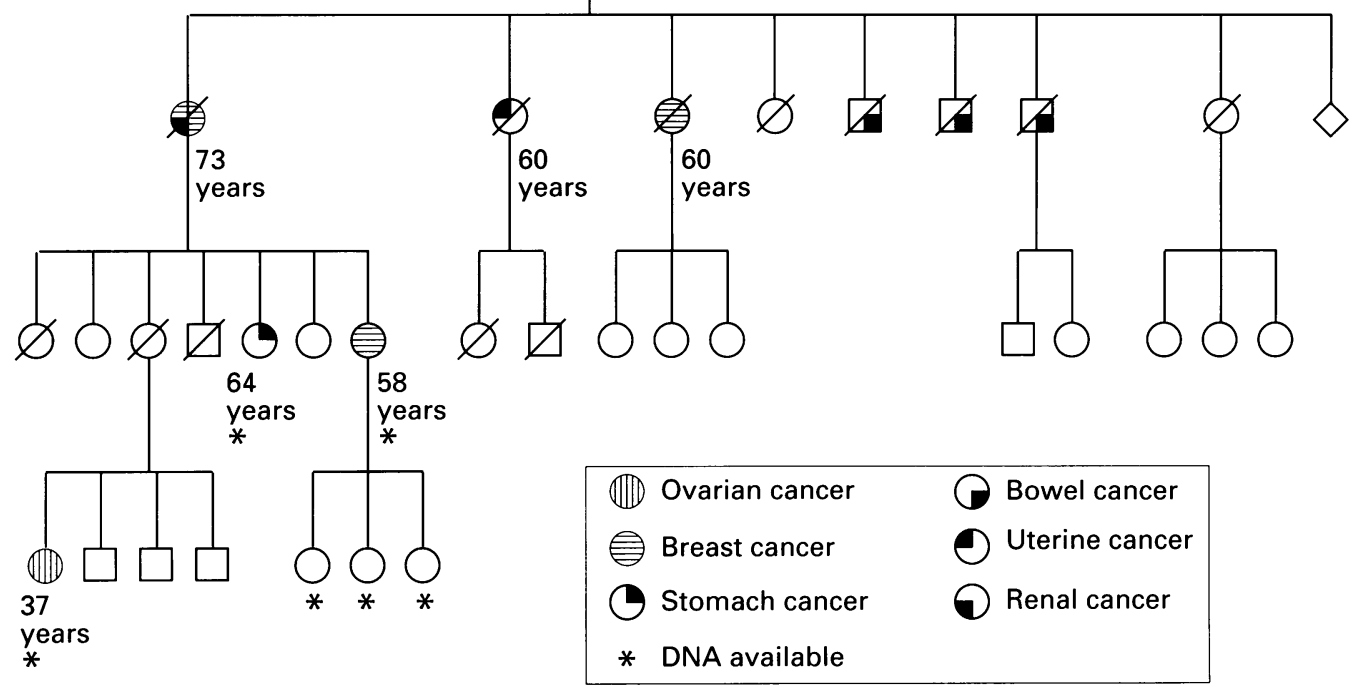

Figure 1 (A) Example of autosomal dominant (AD) pedigree suitable for linkage studies, also containing an example of male transmission. (B) Example of a pedigree suitable for linkage studies but few samples available.

500 000. Of these, 145 subjects from 57 pedigrees had a family history of ovarian cancer; 66 are from one large pedigree (family 1 ) which has been reported previously. ${ }^{910}$ This paper will concentrate on the remaining 79 people (56 pedigrees), but will also describe the use of DNA studies in family 1 .

As can be seen in table 1 , in 20 of these 56 pedigrees ovarian cancer was the main or only cancer identified, while in 25 pedigrees both breast and ovarian cancer were seen. The remaining 11 pedigrees contained cases of ovarian and other cancer types, including examples consistent with the Lynch type II syndrome.

If a family included three or more subjects with a relevant cancer and direct vertical transmission or transmission through a male, the family was considered compatible with autosomal dominant (AD) inheritance. Using these criteria, 17 pedigrees were consistent with $\mathrm{AD}$ inheritance but only one is currently suitable for linkage studies. This was because of a lack of tissue or blood samples for DNA extraction, and also in some cases because of an inability to confirm the diagnosis in some family members (fig 1A, B).

Of the $\mathrm{AD}$ families, one contained six cases of ovarian cancers, 10 families included a combination of breast cancer (either unilateral or bilateral) and at least one ovarian cancer, and the remaining six families contained a combination of ovarian, breast, and other cancers such as testicular or gastrointestinal cancers. One of these six families also contained a subject with Hodgkin's lymphoma (table 2).

Those families not meeting the above criteria for $\mathrm{AD}$ inheritance included 15 families with one or two ovarian cancers, 14 families with breast and ovarian cancers, and the remaining 10 families with ovarian cancer along with breast, gastrointestinal, and cervical cancers, and in two of these families a malignant melanoma was seen (table 3). Seven of the families in this category included two subjects with ovarian cancer (table 4). Levels of lifetime risk 
Table 2 Nature of cancers in the autosomal dominant families

\begin{tabular}{|c|c|c|c|c|c|c|c|c|}
\hline \multirow[t]{2}{*}{ Cancer family } & \multirow{2}{*}{$\begin{array}{l}\text { No of } \\
\text { families }\end{array}$} & \multicolumn{7}{|c|}{ Cancer type } \\
\hline & & Ovarian & Breast & Bilat breast & Testis & Colon & Stomach & Other \\
\hline Ovarian & 1 & 6 & - & - & - & - & - & - \\
\hline Breast/ovarian & 10 & 15 & 21 & 2 & - & - & - & - \\
\hline Ovarian and other & 6 & 10 & 7 & 1 & 4 & 5 & 2 & $1^{*}$ \\
\hline
\end{tabular}

*Hodgkin's lymphoma.

Table 3 Nature of cancers in the non-autosomal dominant families

\begin{tabular}{|c|c|c|c|c|c|c|c|c|}
\hline \multirow[t]{2}{*}{ Cancer family } & \multirow{2}{*}{$\begin{array}{l}\text { No of } \\
\text { families }\end{array}$} & \multicolumn{7}{|c|}{ Cancer type } \\
\hline & & Ovarian & Breast & Bilat breast & Testis & Colon & Stomach & Other \\
\hline Ovarian & 15 & 18 & - & - & - & - & - & - \\
\hline Breast/ovarian & 14 & 14 & 18 & 0 & - & - & - & - \\
\hline Ovarian and other & 10 & 12 & 4 & 0 & 0 & 7 & 2 & $4^{*}$ \\
\hline
\end{tabular}

* Two malignant melanoma and two cervical cancer.

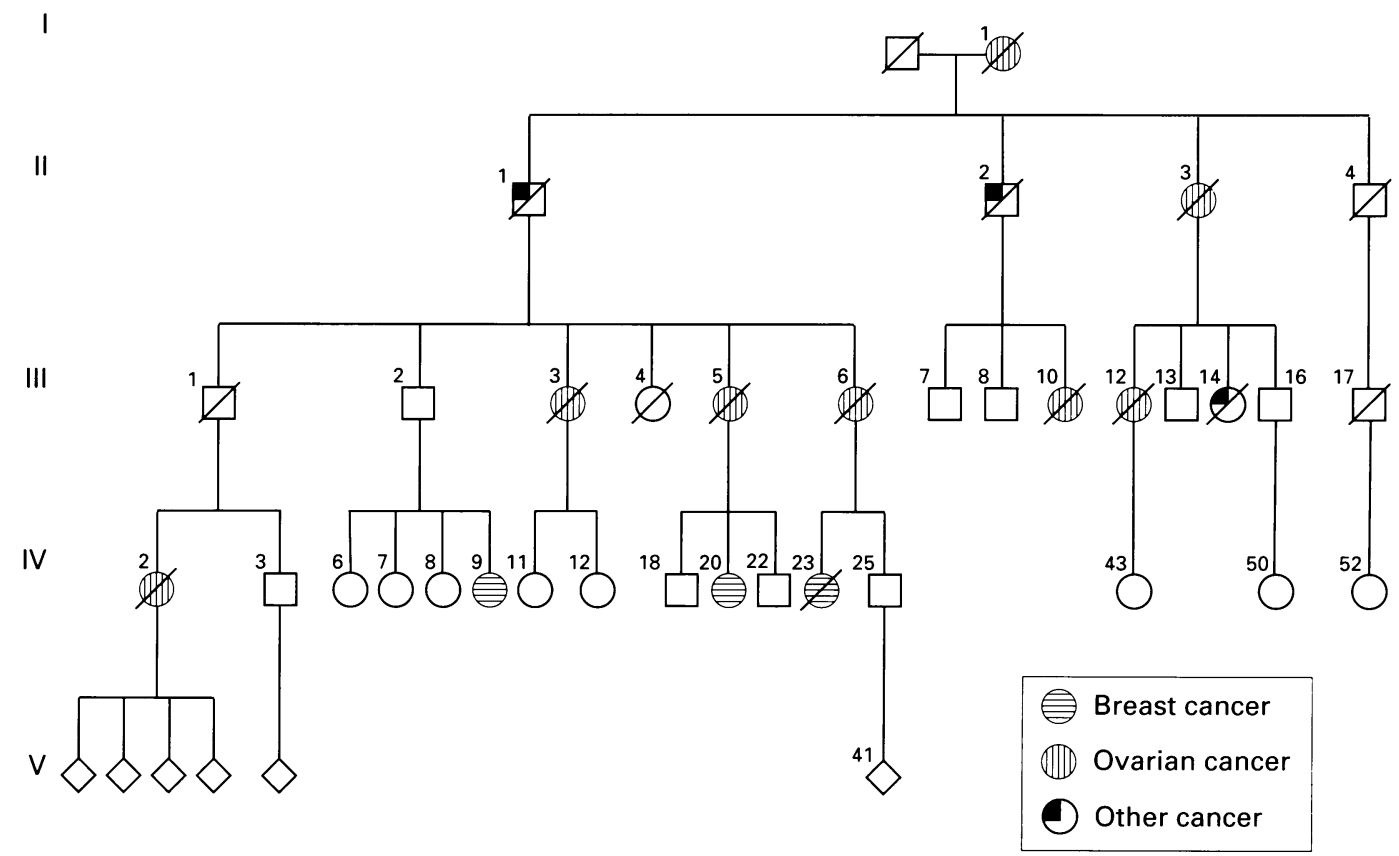

Figure 2 Simplified pedigree of family 1 (linkage studies available).

for these people were calculated as ranging from around $2 \cdot 5 \%$ (one first degree relative $<55$ years affected by ovarian cancer) to around $30 \%$ (two first degree relatives with breast or ovarian cancer) lifetime risk, based on estimations of the probability of inheriting a deleterious gene from relatives affected at different ages. ${ }^{41112}$

Forty-seven people over 18 years of age from family 1 with breast and ovarian cancer syndrome (fig 2, the simplified pedigree of a family previously described by Cruickshank et $a l^{\dagger}$ ) mentioned earlier were counselled on at least two occasions regarding predictive testing by DNA linkage studies, and subsequently requested testing. Only six people in this pedigree (three male and three female) who live in our region declined to attend for counselling. To date, two counselled subjects in that family (one male and one female) have chosen not to have DNA testing. The group who requested testing comprised 29 females and 18 males. Of these, 14 females and 16 males were at $50 \%$ risk of carrying the gene for breast/ovarian cancer, 11 females and two males were at $25 \%$ risk, and four females but no males were at $12.5 \%$ risk (fig $3 \mathrm{~A}$ ). Where the parents were still living, all parents and spouses of those considered to be at risk who were resident in the region attended for counselling and were tested. Therefore no subject was tested whose parent (if unaffected) had not also been tested.

Following testing, these 47 patients were then recategorised as being either at high risk (greater than $95 \%$ probability of carrying the gene based on DNA studies with linked polymorphisms) or low risk (less than $5 \%$ risk of carrying the gene) (fig $3 \mathrm{~B}$ ).

\begin{tabular}{lllc} 
Table 4 & Distribution of ovarian cancers in pedigrees \\
\hline No of ovarian tumours & 3 or more & \multicolumn{1}{c}{2} & \multicolumn{1}{c}{1} \\
\hline $\begin{array}{l}\text { Autosomal dominant pedigree } \\
\text { Non-autosomal dominant }\end{array}$ & 1 & 10 & 6 \\
pedigree & 0 & 7 & 33 \\
\hline
\end{tabular}



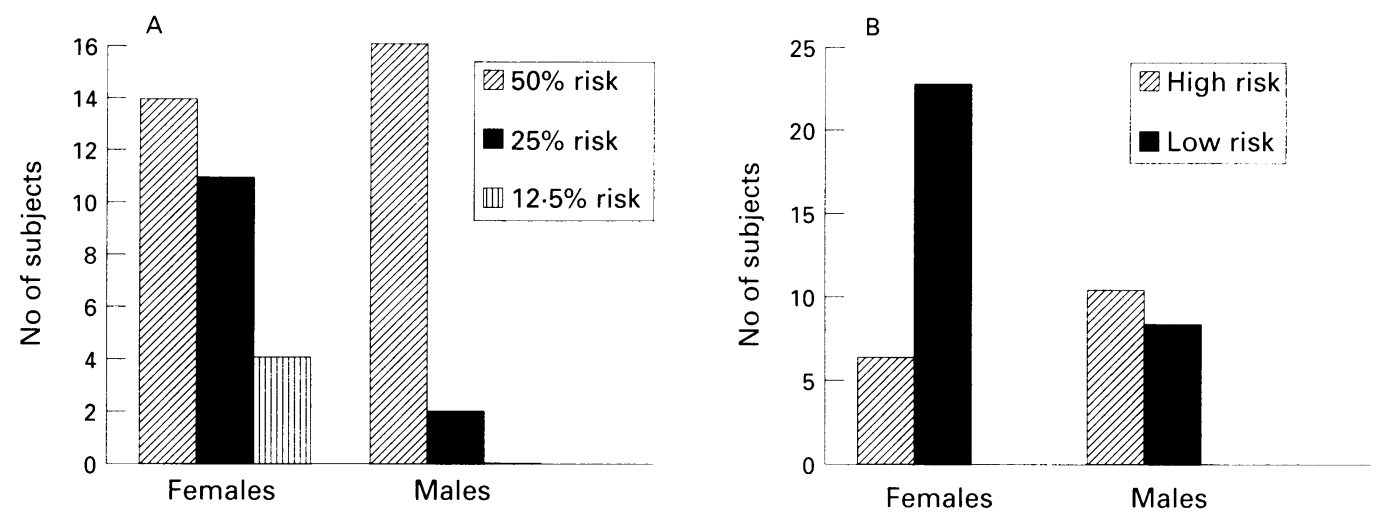

Figure 3 Risk modifications based on DNA studies. (A) Members of family 1 requesting testing. (B) Risk modifications in members of family 1 following testing.

Of the original 29 women, only six were found to be at high risk ( $>95 \%)$ of carrying the predisposing gene. The remaining 23 have been reassured that they are at low risk and once mutation detection can confirm and further refine their low risk status, they may be counselled that they will not require further "high risk" screening. Ten out of 18 males were found to be at high risk of carrying the affected gene. Therefore it is possible to begin appropriate counselling for the female offspring of these high risk males.

Before DNA testing, three females from family 1 opted to have prophylactic mastectomy, and all but one in generation IV who were at risk and with whom contact had been made had prophylactic oophorectomy. After DNA testing, it is too early to say what decisions will be made by those subjects who are at high risk who have not as yet had surgery, based on the results of their DNA tests. Those who are now in the "low risk" category based on these linkage studies appear to be delaying their decisions regarding prophylactic mastectomy, although it is again too early to determine the effect on decisions regarding prophylactic oophorectomy.

\section{Discussion}

A family history which includes ovarian cancer is found in nearly $15 \%$ of "family cancer referrals" to our Genetic Clinic. This constitutes a substantial workload. Few of these patients' pedigrees are suitable for linkage studies, but many would be appropriate for inclusion in studies aimed at detecting mutations in BRCA1.

The implications of a diagnosis of ovarian cancer in a family, especially where there have previously been family members affected with similar cancers, can have a major effect on the lifestyles of other family members. In Aberdeen, we have considerable experience in counselling families with other adult onset diseases such as Huntington's disease, ${ }^{13}$ and the effects that this diagnosis has on other family members. Subjects with a family history of ovarian cancer should receive appropriate counselling regarding their risks of developing an ovarian or related cancer, and screening should be offered if these people are considered to be at high risk. Present management of such patients involves a number of approaches.

\section{LIFESTYLE}

The combined oral contraceptive pill has been found to be protective against ovarian cancer. Inhibition of ovarian function with the combined oral contraceptive pill is associated with a $40 \%$ lower risk of ovarian cancer in the general population. ${ }^{1415}$ However, the effect on the risk in genetically predisposed women is not yet known. The risk of early onset breast cancer in those with a family history of breast cancer taking the combined oral contraceptive pill is as yet unclear, but in women in the general population there is an increase in early onset breast cancer ( $<45$ years) in those taking the combined oral contraceptive pill for more than eight years. ${ }^{16}$ Recent studies have suggested that it is the length of pill use before the first pregnancy which is most relevant. ${ }^{17}$ However, the evidence for use of the combined oral contraceptive pill being a risk factor in breast cancer is less than that of it being protective in ovarian cancer. Therefore in families where there is predominantly a risk of ovarian cancer there may be a balance in favour of combined oral contraceptive usage, particularly as ovarian cancer has a worse prognosis. The oral contraceptive pill may also be the form of contraception of choice, after consideration of other factors such as stability of relationships, contraindications of other forms of contraception in some people, and the risks of an unwanted pregnancy to the physical and mental health of the women.

\section{SCREENING}

At present, women who are referred to the Genetic Clinic in Aberdeen and who are found to be at increased risk of developing ovarian cancer (as defined in the second paragraph of the Introduction to this paper) are offered screening in the form of annual pelvic examination, along with annual transvaginal ultrasound ${ }^{18}$ and serum CA 125 estimation. ${ }^{19} 20$ Any abnormalities that are detected are referred for further follow up and management by a 
gynaecologist with a special interest in gynaecological oncology. This screening policy was adopted as a compromise in Aberdeen, as the effectiveness of the above screening strategies is as yet unproven. While individual centres are attempting to set up protocols for and trials of screening for ovarian cancer in order to ascertain the effectiveness of various forms of surveillance, it is also hoped that in the near future a national trial will begin which will audit the outcome of such screening programmes in women in different at risk categories.

\section{PROPHYLACTIC SURGERY}

Prophylactic bilateral salpingo-oophorectomy may be offered to women over the age of 35 years who have completed their families, after appropriate counselling. ${ }^{2}$ Studies suggest that in such women there is still a residual risk of intra-abdominal carcinomatosis compatible with metastatic ovarian cancer. ${ }^{2122}$ Therefore histological examination of ovaries removed prophylactically is essential as tiny foci of adenocarcinoma may exist.

People who present to our clinic Aberdeen with a family history including ovarian cancer are given a personal risk assessment based on their family history, and if they are thought to be at a high risk of developing breast or ovarian cancer themselves are offered the opportunity of entering our screening programme. If the patient is thought to be a member of an autosomal dominant ovarian cancer family, or at the patient's request, they are offered the opportunity to discuss prophylactic surgery with both a clinical geneticist and a gynaecologist. To date, all those discussed in this paper who are considered to be at high risk of developing ovarian cancer have opted to enter our screening programme.

Following discovery of a strong candidate for the BRCA1 gene, ${ }^{67}$ the four genes relating to $\mathrm{HNPCC}^{23-26}$ and localisation of the BRCA2 $2^{27}$ gene, direct mutation testing may soon be possible in a number of families. Very few of our families would be suitable for linkage as the number of affected subjects for whom samples would be available would be inadequate. However, most families could be submitted for mutation testing for BRCA1 and eventually BRCA2, as in most families blood or tissue samples from many of the affected relatives are available. This may eventually give information regarding the likelihood that it is the segregation of a pathological mutation which is associated with the disease in that family.

When direct mutation testing becomes available, there will be increased demand on clinical and laboratory time. These patients will require pretest counselling, whether or not they opt to proceed to DNA testing. ${ }^{928}$

Predictive testing carries implications for lifestyle similar to other adult onset genetic disorders, such as Huntington's disease ${ }^{28}$ Because of this, it has been suggested that a similar programme of counselling should be available for those at possible high risk of developing an inherited cancer syndrome, for which genetic testing is available. Such a programme proposes that each patient would require at least two one hour pretest counselling sessions and two post-test counselling sessions; the total extra workload for patients referred from the families described here would be around 316 hours. This would comprise 112 hours for those in the $\mathrm{AD}$ category, 180 hours for those not in the $\mathrm{AD}$ category but with at least one first degree relative affected with a relevant cancer, and the remaining 24 hours for those with at least one second degree relative affected with a relevant cancer. This excludes any new referrals both through present channels and those which may result from publicity following the discovery of the BRCAl gene and any other genes. This does not take into account any attempt at family tracing, for counselling of other potentially at risk family members. Conversely, this calculation assumes that tissue samples would be available from affected subjects in all of these families, which may not be the case.

Assuming that up to three such consultations could be carried out per clinic, on present calculations the above workload could occupy a genetic counsellor for the equivalent of 105 to 106 sessions. This excludes the time spent in researching family history and arranging appropriate screening programmes for the consultands.

However, as can be seen from the results of DNA analysis in family 1 mentioned above ${ }^{9}$ (fig 2), more accurate identification of high risk patients can result in fewer people requiring screening or prophylactic surgery. Potential health benefits exist if a decision regarding surgery is postponed because of the unwanted effects of early mastectomy and oophorectomy if these prove to be unnecessary. Therefore DNA analysis can provide reassurance to a majority, and by more accurate targeting of those who require it, associated financial savings for screening and surgical services.

It is interesting to note (from fig 3) that a higher number of men than women were at $50 \%$ risk before testing, and subsequently tested at high risk compared to women, yet there were fewer men than women at $25 \%$ and $12.5 \%$ risk who came forward for testing. It is likely that this higher proportion of males in the $50 \%$ category exists because they have not been "screened out" by actually developing a cancer themselves.

It would be interesting in the future to estimate the cost in terms of genetic testing and counselling and prophylactic surgery, set against costs saved by not screening those who do not carry the mutant allele in this family, but such a study would necessarily be the basis of further research.

1 Heintz APM, Hacker NF, Lagasse LD, et al. Epidemiology and aetiology of ovarian cancer: a review. Obstet Gynecol 1985;66:127-35.

2 Petterson F. Annual report on the results of treatment in gynaecological cancer. Int $\mathcal{f}$ Gynaecol Obstet 1991;
36(suppl):238-77.

3 Lynch HT, Albano WA, Lynch JF, et al. Surveillance and management of patients at high genetic risk for ovarian cancer. Obstet Gynecol 1982;59:589-96.

4 Ponder BAJ, Easton DF. Risk of ovarian cancer associated with a family history: preliminary report of the OPCS study. In: Sharp F, Mason WP, Leake RE, eds. Ovarian 
cancer - biological and therapeutic challenges. London: Chapman \& Hall Medical, 1989:3-6.

5 Evans DGR, Ribiero G, Warrell D, et al. Ovarian cancer family and prophylactic choices. F Med Genet 1992;29: $416-8$

6 Miki Y, Swensen J, Shattuck-Eidens D, et al. A strong candidate for the breast and ovarian cancer susceptibility gene BRCA1. Science 1994;266:66-71.

7 Futreal PA, Liu Q, Shattuck-Eidens D, et al. BRCA1 mutations in primary breast and ovarian carcinomas. Science ations in primary

8 Eeles R, Ponder B, Easton D, et al. Clinical protocol for 17q predictive testing in breast/ovarian families. Draft protocol for consideration by CFSG, December 1993.

9 Cruickshank DJ, Haites N, Anderson S, et al. The multidisciplinary management of a family with epithelial ovarian cancer. Br f Obstet Gynaecol 1992;99:226-31. 10 Milner BJ, Allan LA, Kelly KF, et al. Linkage studies with
$17 \mathrm{q}$ and $18 \mathrm{q}$ markers in a breast/ovarian cancer family. Am f Hum Genet 1993;52:761-6.

11 Ponder B. Non-surgical aspects of ovarian cancer. Report of a Meeting of Physicians and Scientists, Institute of Cancer Research and the Royal Marsden Hospital, London. Lancet 1994;343:335-40.

12 Murday V. Genetic counselling in the cancer family clinic. Eur f Cancer 1994;30A:2015-29.

13 Simpson SA, Besson J, Alexander D, et al. One hundred requests for predictive testing for Huntington's disease. Clin Genet 1992;41:326-30.

14 Newhouse ML, Pearson RM, Fullerton JM, et al. A case study of carcinoma of the ovary. Br f Prev Soc Med 1977 $31: 148-52$.

15 Cancer and Steroid Hormone Study Group. The reduction in risk of ovarian cancer associated with oral contraceptive use. $N$ Engl f Med 1987;316:650-5.

16 UK National Cancer Control Study Group. Oral contraceptive use and breast cancer risk in young women. Lancet 1989;i:973-82.
17 Malone KE, Daling JR, Weiss NS. Oral contraceptives in relation to breast cancer. Epidemiol Rev 1993;15:80-97.

18 Bourne TH, Campbell S, Reynolds KM, et al. Screening for early familial ovarian cancer with transvaginal ultrasonography and colour blood flow imaging. BMF 1993; 306: 1025-9.

19 Einhorn N, Sjovall K, Knapp RC, et al. Prospective evaluation of serum CA125 levels for early detection of ovarian cancer. Obstet Gynecol 1992;80:14-18.

20 Jacobs I, Prys Davies A, Bridges J, et al. Prevalence screening for ovarian cancer in post-menopausal women by CA125 measurement and ultrasonography. $B M F$ 1993;306:10304.

21 Tobacman JK, Green MH, Tucker MA, et al. Intra-abdominal carcinomatosis after prophylactic oophorectomy in ovarian cancer-prone families. Lancet 1982;ii:795-7.

22 Chen KTK, Schooley JL, Flam MS. Peritoneal carcinomatosis after prophylactic oophorectomy in familial ovarian cancer syndrome. Obstet Gynecol 1985;66(suppl 3):93-4S

23 Peltomaki P, Aaltonen LA, Sistonen P, et al. Genetic mapping of a locus predisposing to human colorectal cancer Science 1993;260:810-12

24 Fishel R, Lescoe MK, Rao MRS, et al. The human mutator gene homolog $M S H 2$ and its association with hereditary nonpolyposis colon cancer. Cell 1993;75:1027-38.

25 Leach FS, Nicolaides NC, Papadopoulos N, et al. Mutations of a mut $S$ homology in hereditary nonpolyposis colorectal of a mut $S$ homology in hereditary

26 Papadopolous N, Nicolaides NC, Nei YF, et al. Mutation of a mutL homolog in hereditary colon cancer. Science 1994;263:1625-9.

27 Wooster R, Neuhausen S, Mangion J, et al. Localisation of a breast cancer susceptibility gene BRCA2 to chromosome 13q 12-13. Science 1994;265:2088-90.

28 Simpson SA, Dean JCS, Haites NE Genetic prediction of adult onset disease. BMF 1994;308:535. 